International Journal of Pure and Applied Mathematics

Volume 94 No. 2 2014, 155-161

ISSN: 1311-8080 (printed version); ISSN: 1314-3395 (on-line version)

url: http://www.ijpam.eu

doi: http://dx.doi.org/10.12732/ijpam.v94i2.3

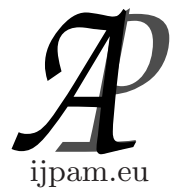

\title{
A NOTE ON PERFECT FUZZY MATCHING
}

\author{
R. Seethalakshmi ${ }^{1}$, R.B. Gnanajothi ${ }^{2}$ \\ ${ }^{1}$ Saiva Bhanu Kshatriya College \\ Aruppukottai, 626 101, Tamilnadu, INDIA \\ ${ }^{2}$ V.V. Vanniaperumal College \\ Virudhunagar, 626 001, Tamilnadu, INDIA
}

\begin{abstract}
The notion of matching in a fuzzy graph could be defined using the concept of effective edges [8] or by fractional matching [4]. In this paper, we derive a necessary condition for a fuzzy graph on a cycle or a complete graph or a stargraph to have a perfect fuzzy matching. Also we discuss perfect fuzzy matching on strong regular fuzzy graphs.
\end{abstract}

AMS Subject Classification: 05C72, 03E72

Key Words: fuzzy graph, fuzzy matching, perfect fuzzy matching

\section{Introduction}

Zadeh introduced the notion of Fuzzy sets and Fuzzy relations to deal with the problems of uncertainty in real physical world. In 1975, Rosenfeld [5] introduced the concept of fuzzy graphs. Using the concept of effective edges, Somasundaram [8] defined matching in a fuzzy graph. This matching is defined only for those graphs having effective edges. Ramakrishnan P.V and Vaidyanathan M [4] introduced matching in a fuzzy graph using the concept of fractional matching. The notion of fractional matching given by Scheinerman [1], in 1997, involves the vertex weight, the edge weight and the incidence of

Received: December 20, 2013

(C) 2014 Academic Publications, Ltd.

${ }^{\S}$ Correspondence author url: www.acadpubl.eu 
edges. In this paper, we derive the necessary condition for the fuzzy graph on a cycle or a complete graph or a star graph to have perfect fuzzy matching. Further, we prove that a strong regular fuzzzy graph will have no perfect fuzzy matching.

\section{Preliminaries}

Definition 2.1. A fuzzy graph $G=(\sigma, \mu)$ is a pair of functions $\sigma: V \rightarrow$ $[0,1]$ and $\mu: V \times V \rightarrow[0,1]$ with $\mu(u, v) \leq \sigma(u) \wedge \sigma(v), \forall u, v \in V$, where $V$ is a finite nonempty set and $\wedge$ denote minimum.

Definition 2.2. A fuzzy graph $G=(\sigma, \mu)$ is defined to be a strong fuzzy graph if

$\mu(u, v)=\sigma(u) \wedge \sigma(v), \forall(u, v) \in E$.

Definition 2.3. A fuzzy graph $G=(\sigma, \mu)$ is defined to be a complete fuzzy graph if

$\mu(u, v)=\sigma(u) \wedge \sigma(v), \forall u, v \in V$.

Definition 2.4. Let $G=(\sigma, \mu)$ be a fuzzy graph on $G^{*}=(V, E)$. The fuzzy degree of a node $u \in V$ is defined as $(f d)(u)=\sum_{u \neq v, v \in V} \mu(u, v)$.

$G$ is said to be regular fuzzy graph if each vertex has same fuzzy degree.

If $(f d)(v)=k, \forall v \in V, G$ is said to be $k$-regular fuzzy graph.

Definition 2.5. Let $G=(\sigma, \mu)$ be a fuzzy graph on $(V, E)$. where $V$ is the vertex set and $E$ is the set of edges with non-zero weights. A subset $M$ os $E$ is called a Fuzzy matching if for each vertex $u$, we have $\sum_{v \in V} \mu(u, v) \leq \sigma(u)$.

Example 2.6. Let $G=(\sigma, \mu)$ be a fuzzy graph on $(V, E)$ where $V=\left\{v_{1}, v_{2}, v_{3}, v_{4}\right\}$ and $E=\left\{e_{1}, e_{2}, e_{3}, e_{4}\right\}$ with $e_{1}=v_{1} v_{2}, e_{2}=v_{2} v_{3}, e 3=$ $v_{3}, v_{4}$ and $e_{4}=v_{4} v_{1}$

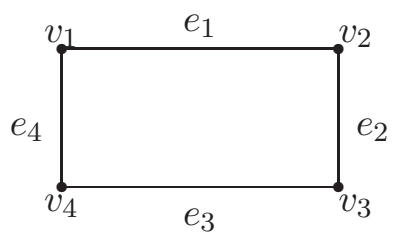

$$
\begin{aligned}
& \sigma\left(v_{1}\right)=1, \sigma\left(v_{2}\right)=0.5, \sigma\left(v_{3}\right)=0.4, \sigma\left(v_{4}\right)=0.7 \\
& \mu\left(e_{1}\right)=0.3, \mu\left(e_{2}\right)=0.4, \mu\left(e_{3}\right)=0.2, \mu\left(e_{4}\right)=0.5 \\
& \sum_{v_{2} \in V,\left(v_{1}, v_{2}\right) \in M} \mu\left(v_{1}, v_{2}\right)=0.3+0.5=0.8 \leq 1=\sigma\left(v_{1}\right) .
\end{aligned}
$$




$$
\begin{aligned}
& \sum_{v_{3} \in V,\left(v_{2}, v_{3}\right) \in M} \mu\left(v_{2}, v_{3}\right)=0.4+0.3=0.7 \leq 0.5=\sigma\left(v_{2}\right) . \\
& \sum_{v_{1} \in V,\left(v_{1}, v_{4}\right) \in M} \mu\left(v_{1}, v_{4}\right)=0.5+0.2=0.7 \leq 0.7=\sigma\left(v_{4}\right) .
\end{aligned}
$$

Thus $M=\left\{e_{1}, e_{2}, e_{4}\right\}$ is a fuzzy matching in $G$.

Definition 2.7. A fuzzy matching $M$ is called a Perfect Fuzzy Matching if for each vertex $u, \sum_{v \in V,(u, v) \in M} \mu(u, v)=\sigma(u)$.

Example 2.8. Let $G=(\sigma, \mu)$ be a fuzzy graph on $(V, E)$ where $V=\left\{v_{1}, v_{2}, v_{3}, v_{4}\right\}$ and $E=\left\{e_{1}, e_{2}, e_{3}, e_{4}, e_{5}\right\}$ with $e_{1}=v_{1} v_{2}, e_{2}=v_{2} v_{3}, e 3=$ $v_{3}, v_{4}, e_{4}=v_{4} v_{1}$ and $e_{5}=v_{4} v_{2}$

$$
\begin{aligned}
& \sigma\left(v_{1}\right)=1, \sigma\left(v_{2}\right)=0.9, \sigma\left(v_{3}\right)=0.8, \sigma\left(v_{4}\right)=0.9 \\
& \mu\left(e_{1}\right)=0.4, \mu\left(e_{2}\right)=0.5, \mu\left(e_{3}\right)=0.3, \mu\left(e_{4}\right)=0.6, \mu\left(e_{5}\right)=0.8 \\
& \text { Here } \sum_{v_{2} \in V,\left(v_{1}, v_{2}\right) \in M} \mu\left(v_{1}, v_{2}\right)=0.4+0.6=1=\sigma\left(v_{1}\right) . \\
& \sum_{v_{3} \in V,\left(v_{2}, v_{3}\right) \in M} \mu\left(v_{2}, v_{3}\right)=0.4+0.5=0.9=\sigma\left(v_{2}\right) . \\
& \sum_{v_{3} \in V,\left(v_{3}, v_{4}\right) \in M} \mu\left(v_{3}, v_{4}\right)=0.5+0.3=0.8=\sigma\left(v_{3}\right) . \\
& \sum_{v_{4} \in V,\left(v_{1}, v_{4}\right) \in M} \mu\left(v_{1}, v_{4}\right)=0.3+0.6=0.9=\sigma\left(v_{4}\right) .
\end{aligned}
$$

Thus $M=\left\{v_{1} v_{2}, v_{2} v_{3}, v_{3} v_{4}, v_{4} v_{1}\right\}$ is a perfect fuzzy matching in $G$.

Definition 2.9. Let $G=(\sigma, \mu)$ be a fuzzy graph and $M$ be a fuzzy matching. Then fuzzy matching number $\Gamma(G)$ is defined to be $\Gamma(G)=$ $\sum_{(u, v) \in M} \mu(u, v)$.

Example 2.10. In example 2.6, $\Gamma(G)=1$.2. In example 2.8, $\Gamma(G)=1.8$.

Theorem 2.11. [6] Let $G=(\sigma, \mu)$ be a fuzzy graph on $K_{n, n}$ with bipartition $(X, Y)$ where $X=\left\{u_{1}, u_{2}, \cdots, u_{n}\right\}$ and $Y=\left\{v_{1}, v_{2}, \cdots, v_{n}\right\}$. Then $G$ is strong regular if and only if

1. $\sigma\left(v_{j}\right)=k$

2. $\sigma\left(u_{i}\right)=k$.

3. $\mu\left(u_{i}, v_{j}\right)=k$, for $i, j=1,2, \cdots n$ and for some $k$.

\section{Perfect Fuzzy Matching}

In this section we discuss some necessary conditions for some of the fuzzy graphs to have a perfect matching.

Theorem 3.1. Let $G=(\sigma, \mu)$ be a regular fuzzy graph on the cycle $(V, E)$. If $\sigma(u)=k$, which is constant for all $u \in V$ and $\mu(u, v)=\frac{k}{2}$ for all $(u, v) \in E$, then $E$ is a perfect fuzzy matching for $G$. 


\section{Proof}

Since only two edges are incident wtih each vertex for cycles, for any vertex $v \in V$

$$
\begin{aligned}
\sum_{v \in V,(u, v) \in E} \mu(u, v) \text { where } v, w \in V & =\mu(u, v)+\mu(u, w) \\
& =\frac{k}{2}+\frac{k}{2} \\
& =k=\sigma(u)
\end{aligned}
$$

Then $E$ is a perfect fuzzy matching in $G$.

The converse of the above theorem need not be true. This can be seen using the following example.

Example 3.2. Let $G=(\sigma, \mu)$ be a fuzzy graph on $(V, E)$ where $V=\left\{v_{1}, v_{2}, v_{3}, v_{4}\right\}$ and $E=\left\{e_{1}, e_{2}, e_{3}, e_{4}\right\}$ where $e_{1}=v_{1} v_{2}, e_{2}=v_{2} v_{3}, e 3=$ $v_{3}, v_{4}$ and $e_{4}=v_{4} v_{1}$

Define $\sigma\left(v_{1}\right)=0.6, \sigma\left(v_{2}\right)=0.6, \sigma\left(v_{3}\right)=0.5, \sigma\left(v_{4}\right)=0.5$ and

$\mu\left(e_{1}\right)=0.4, \mu\left(e_{2}\right)=0.2, \mu\left(e_{3}\right)=0.3, \mu\left(e_{4}\right)=0.2$.

Then the graph $G$ has perfect fuzzy matching but the conditions of the above theorem are not satisfied.

Theorem 3.3. Let $G=(\sigma, \mu)$ be a complete fuzzy graph $K_{n}$ on $(V, E)$. If $\sigma(u)=k$ which is constant for all $u \in V$ and $\mu(u, v)=\left[\frac{k}{n}\right]=k_{1}$, for all $(u, v)$ on the cycle $C_{n}$ and $\mu(u, v)=\frac{k-2 k_{1}}{n-3}$ for all the interior edges $(u, v) \in E$, then $E$ is a perfect fuzzy matching for $G$.

\section{Proof}

For any complete fuzzy graph $K_{n}$, two edges are incident with each vertex of the cycle and remaining $(n-3)$ edges are incident with interior vertices. Hence

$$
\begin{aligned}
\sum_{v \in V,(u, v) \in E} \mu(u, v) & =2 k_{1}+(n-3)\left(\frac{k-2 k_{1}}{n-3}\right. \\
& =2 k_{1}+k-2 k_{1} \\
& =k \\
& =\sigma(u), \text { for each vertex } u .
\end{aligned}
$$

Therefore $E$ is a perfect fuzzy matching for $G$.

The converse of the above theorem is not true. This can be seen using the following example. 
Example 3.4. Let $G=(\sigma, \mu)$ be a fuzzy graph on $(V, E)$ where $V=$ $\left\{v_{1}, v_{2}, v_{3}, v_{4}\right\}$ and $E=\left\{e_{1}, e_{2}, e_{3}, e_{4}, e_{5}, e_{6}\right\}$

where $e_{1}=v_{1} v_{2}, e_{2}=v_{2} v_{3}, e 3=v_{3}, v_{4}, e_{4}=v_{4} v_{1}, e_{5}=v_{4} v_{2}$ and $e_{6}=v_{1} v_{3}$

Define $\sigma\left(v_{1}\right)=0.9, \sigma\left(v_{2}\right)=0.8, \sigma\left(v_{3}\right)=0.7, \sigma\left(v_{4}\right)=0.8$ and

$\mu\left(e_{1}\right)=0.2, \mu\left(e_{2}\right)=0.3, \mu\left(e_{3}\right)=0.1, \mu\left(e_{4}\right)=0.4, \mu\left(e_{5}\right)=0.3, \mu\left(e_{6}\right)=0.3$.

Then $E$ is a perfect matching for $G$ but the conditions of the above theorem are not satisfied.

Theorem 3.5. Let $G=(\sigma, \mu)$ be a strong fuzzy graph on the star graph $S_{n}=(V, E)$ with $V=\left\{v, v_{1}, v_{2}, \cdots, v_{n-1}\right\}$. If $\sigma\left(v_{i}\right)=k, \forall i=1,2, \cdots,(n-1)$ and if $\sigma(v)=(n-1) k$, then $E$ is a perfect fuzzy matching in $G$.

\section{Proof}

Since $G$ is strong,

$$
\begin{aligned}
\mu\left(v, v_{i}\right) & =\sigma(v) \wedge \sigma\left(v_{i}\right), \forall v_{i} \in V \\
& =(n-1) k \wedge k \\
& =k
\end{aligned}
$$

Now, for any $v \in V$,

$$
\begin{aligned}
\sum_{v_{i} \in V,\left(v, v_{i}\right) \in E} \mu\left(v, v_{i}\right) & =\sum_{\left(v, v_{i}\right) \in E} k \\
& =(n-1) k, \text { since }(n-1) \text { edges are incident with } v \\
& =\sigma(v)
\end{aligned}
$$

Therefore, $E$ is a perfect fuzzy matching for $G$.

The converse of the above theorem is also not true. This can be seen from the following example.

Example 3.6. Let $G=(\sigma, \mu)$ be a fuzzy graph on $(V, E)$ where $V=$ $\left\{v_{1}, v_{2}, v_{3}, v_{4}, v_{5}\right\}$ and $E=\left\{e_{1}, e_{2}, e_{3}, e_{4}, e_{5}\right\}$ where $e_{1}=v v_{1}, e_{2}=v v_{2}, e_{3}=$ $v v_{3}, e_{4}=v v_{4}$ and $e_{5}=v v_{5}$.

Define $\sigma\left(v_{1}\right)=0.2, \sigma\left(v_{2}\right)=0.1, \sigma\left(v_{3}\right)=0.2, \sigma\left(v_{4}\right)=0.1, \sigma\left(v_{5}\right)=$ $0.2, \sigma(v)=0.8$

$\mu\left(e_{1}\right)=0.2, \mu\left(e_{2}\right)=0.1, \mu\left(e_{3}\right)=0.2, \mu\left(e_{4}\right)=0.1, \mu\left(e_{5}\right)=0.2$.

Then $E$ is a perfect matching for $G$ but the conditions of the above theorem are not satisfied.

The following theorem establishes that a strong regular fuzzy graph need not have a perfect fuzzy matching. 
Theorem 3.7. If $G=(\sigma, \mu)$ is a strong regular fuzzy graph on $(V, E)$ with each vertex is of degree atleast two, then $E$ is not a perfect fuzzy matching in G.

Proof If possible, let $E$ be a perfect fuzzy matching for $G$. Then

$\sum_{v \in V,(u, v) \in E} \mu(u, v)=\sigma(u), \forall u \in V, \longrightarrow(1)$ by definition.

Since $G$ is regular, $(f d)(u)=$ constant $=k$ (say), $\forall u \in V$.

Therefore, $\sum_{v \in V,(u, v) \in E} \mu(u, v)=k, \forall u \in V$, which imply

$\sigma(u)=k, \forall u \in V$. ( Using (1)).

Now from (1) $\sum_{v \in V,(u, v) \in E} \mu(u, v)=k \Rightarrow \mu(u, v)<k$, since atleast two edges incident with $u$, for some $(u, v)$.

Since $G$ is strong, $\sigma(u) \wedge \sigma(v)<k \Rightarrow k \wedge k<k \Rightarrow k<k$, which is a contradiction.

Therefore, $E$ is not perfect fuzzy matching for $G$.

In particular, we have the following

Corollary 3.8. If $G=(\sigma, \mu)$ is strong regular fuzzy graph on $K_{n, n}$, then $E$ is not a perfect fuzzy matching.

Proof Suppose $E$ is a perfect fuzzy matching for $G$.

Then $\sum_{v \in V,(u, v) \in E} \mu(u, v)=\sigma(u)$, for each $u \in V$.

Let $\sigma(u)=k, \forall u \in X$.

Therefore, $\sum_{v \in V} \mu(u, v)=k, \forall u \in X$.

Hence $\mu(u, v)<k$, for some $v \in V$, which is a contradiction to theorem 2.11 Therefore, $E$ is not a perfect fuzzy matching for $G$.

\section{References}

[1] Edward R. Scheinerman, Daniel H and Ullman, Fractional Graph Theory, John Wiley and sons Inc 1997

[2] Morderson, J.N, Nair, P.S, Fuzzy Graphs and Fuzzy Hyper Graphs, Physics - verlag, Heidelberg (2000)

[3] Nagoorgani, A and Chandrasekaran V.T, A First Look at Fuzzy Graph $\operatorname{Theory}(2010)$

[4] Ramakrishnan P.V and Vaidyanathan M, Matching in Fuzzy graphs. Proceedings of National Conference NCDMA 2007.

[5] Rosenfeld A, Fuzzygraphs In : Zadeh, L.A. Fu, K.S.Shimura, M (Eds), Fuzzy sets and their Applications (Academic Press, Newyark). 
[6] Seethalakshmi R and Gnanajothi R.B, On Strong Regular Fuzzy graph, Proceedings of the Heber International Conference on Applications of Mathematics and Statistics, Jan 2010, pp.364-369.

[7] Shimura M (Eds.), Fuzzy sets and their applications, Acadamic Press, New York, pp 77-95, 1975.

[8] Somasundaram A, Somasundaram S, Domination in Fuzzy graphs, I. Pattern Recognition letters 19, 1998, pp 787-791.

[9] Zadeh L.A. Similarity relations and fuzzy ordering Information sciences 971; 3(2): 177-200. 
